\title{
Towards quality by design in pharmaceutical manufacturing: modelling and control of air jet mills
}

\author{
Satyajeet Bhonsale ${ }^{1,2}$, Dries Telen ${ }^{1}$, Bard Stokbroekx ${ }^{2}$, and Jan Van Impe ${ }^{1 *}$ \\ ${ }^{1}$ BioTeC $+\&$ OPTEC, Department of Chemical Engineering, KU Leuven, Ghent, Belgium \\ ${ }^{2}$ Crystallization Technology Unit, Janssen Pharmaceutica, Beerse, Belgium
}

\begin{abstract}
Milling is an important step in pharmaceutical manufacturing as it not only determines the final formulation of the drug product, but also influences the bioavailability and dissolution rate of the active pharmaceutical ingredient (API). In this respect, the air jet mill (AJM) is most commonly used in the pharmaceutical industry as it is a non-contaminating and non-degrading self-classifying process capable of delivering narrow particle size distributions (PSD). Keeping the principles of Quality by Design in mind, the Critical Process Parameters (CPPs) of the AJM have been identified to be the pressures at the grinding nozzles, and the feed rate which affect the PSD, surface charge and the morphology of the product (i.e. the Critical Material Attributes (CMAs)). For the purpose of this research, the PSD is considered to be the only relevant CMA. A population balance based model is proposed to simulate the dynamics milling operation by utilizing the concept of breakage functions. This model agrees qualitatively with experimental observations of the air jet mill unit present at Janssen Pharmaceutica but further steps for model validation need to be carried out.
\end{abstract}

\section{Introduction}

The air jet mill (AJM) is one of the most common equipments used to micronize active pharmaceutical ingredients (API) in the pharmaceutical industry. The huge popularity of the jet mill arises from the fact that it does not involve any moving parts, thus leading to a noncontaminating size reduction process. The primary cause of breakage in the air jet mill is the particle-particle impact along with the particle-wall impact. Pressurized carrier gas is injected tangentially through the nozzles placed around the chamber generating a high speed fluid vortex in the chamber. This vortex has been visualized with help of computer simulations [1] and also experimentally [2]. The material, introduced into the mill by air pushers exploiting the venturi effect, falls into this fluid vortex and is accelerated to (super)sonic velocities (depending on the type of nozzle used). The particles then break through multiple impacts with the wall and other particles. As the dependency of centrifugal force on the particle size $\left(\sim \mathrm{x}^{3}\right)$ is much stronger than that of the drag force $\left(\sim \mathrm{x}^{2}\right)$, the breaking particles experience much more reduction in centrifugal force than in drag force. Thus, as the particles break, they move more and more towards the centre until, at one specific particle size range (which depends on the operating conditions used) the drag force exceeds the centrifugal force causing the particles to exit the grinding chamber. Thus, the AJM is considered to be a selfclassifying mill.

Corresponding author: jan.vanimpe@kuleuven.be

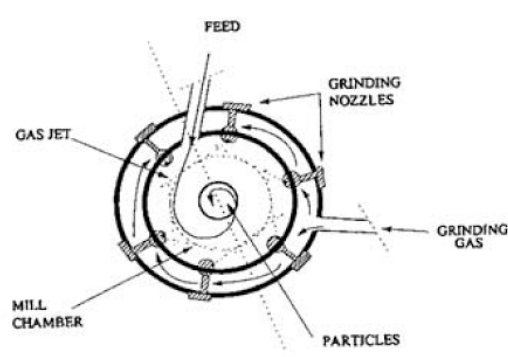

Figure 1. Schematics of the Air Jet Mill [1]

Despite its extensive use, relatively few studies can be found in the literature which deal with modelling or control of the air jet mills, especially for organic compounds. A comprehensive modelling exercise on air jet mills was carried out by Gommeren et al. [3], however, a specially designed jet mill was used which is not realistic in an industrial environment. The parameters in this model are difficult to identify based on experimental data. Moreover, not enough simulation studies were presented to get a comprehensive idea of the model. Some other studies have been aimed at modelling the air jet mill using Markovian theory [4], or steady state population balance model [5], however these are not directed towards control. The output of the mill depends not only on the operating conditions but also on the material characteristics. Considering that every material behaves differently, modelling of milling processes becomes challenging. The present research aims at developing a robust and efficient model for the AJM which could in the 
future be utilized to implement a control strategy to achieve the required particle size distribution (PSD).

\section{Mathematical Description}

Attempts to model milling processes have been made using various techniques like discrete element modelling (DEM) [6], fracture mechanics based modelling [7], population balance modelling (PBM) $[3,5]$, etc. In this work, the PBM framework is utilized to obtain a model for the air jet mill. PBMs have been widely and successfully used in modelling particulate processes like crystallization, milling, mixing [8-9], etc. For the breakage process in consideration, a size-discrete population balance model has been developed. The bases of using a PBM for comminution are the concepts of breakage rate and breakage distribution function which were first proposed by Epstein [10]. The breakage rate $\left(k_{i}\right)$ defines the rate at which the particles in the size class $i$ break under loading conditions. The breakage distribution function $\left(b_{i j}\right)$ defines the probability that a particle breaking from size class $j$ will fall in size class $i$. The breakage rate is commonly described as [11]

$$
k_{i}=\alpha\left(\frac{x_{i}}{x_{1}}\right)^{\gamma}
$$

where $\alpha$ and $\gamma$ are fitting parameters, $x_{i}$ is the volumetric mean size of the class $i$, and $x_{1}$ is the largest size class. $\alpha$ and $\gamma$ are the fitting parameters. For milling processes with small time scales, the breakage distribution function can be approximated as [12]

$$
b_{i j}=\frac{k_{i-1}-k_{i}}{k_{j}}
$$

As a jet mill is a self-classifying mill, the output of the mill has a different particle size distribution than that of the material in hold-up. To simulate the internal classification, the concept of Tromp curve is used. It is assumed that the Tromp curve follows a log-normal distribution $[3,13]$ with respect to the particle size and in its simplest form can be defined as

$$
P_{i}=\frac{1}{1+e^{K\left(x_{i}-x_{50}\right)}}
$$

where $K$ and $x_{50}$ are the fitting parameters. It is obvious that all the parameters in the model will be influenced by the operating parameters of the mill, namely, solid feed rate and grinding nozzle pressure. Moreover, under dynamic conditions the hold-up inside the mill will increase with time until it reaches a steady state value which is when the mill is said to have reached a stable zone. This hold up will also depend on the operating conditions and will affect the parameters in the model. However, for the purposes of this preliminary simulation study, the parameters are assumed to be constant. Unlike the Gommeren's model, the proposed model does not compartmentalize the mill and an overarching PBM is written over the entire mill. This helps reducing the number of parameters which need to be estimated from experimental studies.

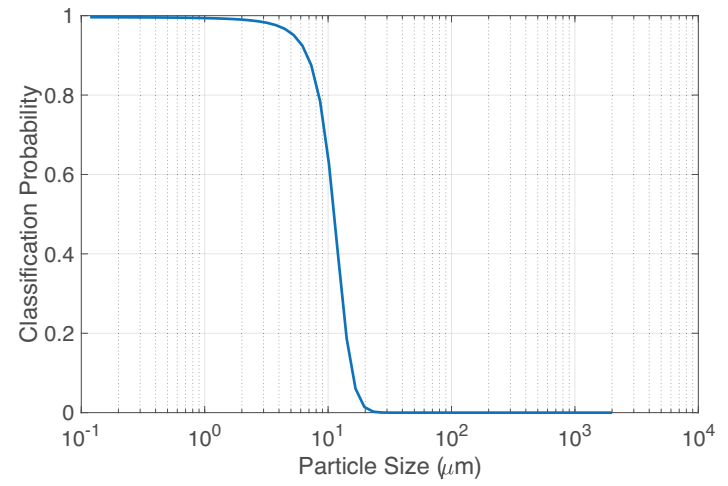

Figure 2. Classification probability as a function of particle size

The population balance model is now described as follows

$$
\begin{gathered}
\frac{d m_{i}}{d t}=f_{\text {in }, i} \Phi_{\text {in }}-P_{i} m_{i}- \\
\Phi_{i} m_{i}+\sum_{j=1}^{i} b_{i j} k_{j} m_{j} \\
=\sum P_{i} m_{i} \\
f_{\text {out }, i}=\frac{P_{i} m_{i}}{\Phi_{\text {out }}}
\end{gathered}
$$

where, $m_{i}$ is the mass of the particles in size class $i$ within the milling chamber. The total mass holdup inside the chamber is then given by $\sum m_{i} . f_{i n, i}$ is the particle size distribution of the feed ( $\%$ in size class $i$ ), $\Phi_{i n}$ is the solid feed rate to the mill, $\Phi_{\text {out }}$ is the total production rate of the mill, and $f_{\text {out }, i}$ is the particle size distribution of the output. In typical experimental conditions, the solid feed rate and the particle size distribution of the feed are known, while the output particle size distribution is measured. The hold-up inside the mill and the product output rate, however, cannot be measured directly leading to identifiability issues as will be discussed later.

Table 1. Parameter values used for simulation

\begin{tabular}{|c|c|}
\hline Parameter & Value \\
\hline$\alpha$ & 5 \\
\hline$\gamma$ & 0.9596 \\
\hline $\mathrm{K}$ & 0.5 \\
\hline $\mathrm{X}_{50}$ & 11.2 \\
\hline$\Phi_{\text {in }}$ & $10(\mathrm{~g} / \mathrm{s})$ \\
\hline
\end{tabular}

\section{Simulation Study}

As previously mentioned, the breakage parameters of the jet mill depend on the process conditions and in turn on the mass hold up within the milling chamber. The 
classification of particles within this mill are also affected by these conditions. In this preliminary analysis, timeconstant parameters have been used with values mentioned in Table 1. Figure 2 depicts the classification curve when using the parameters defined in Table 1.

For this simulation, the 61 size classes have been considered. The largest size class $(i=1)$ has the value $2000 \mu \mathrm{m}$, and a geometric progression with ratio of 1.1795 is used to define all the other size classes. The motivation for the number and values is that the particle size distribution measured by the Insitec online laser diffraction unit (Malvern Instruments Ltd.) is based these values. This will enable the direct introduction of measured data for parameter estimation and model validation in the future.

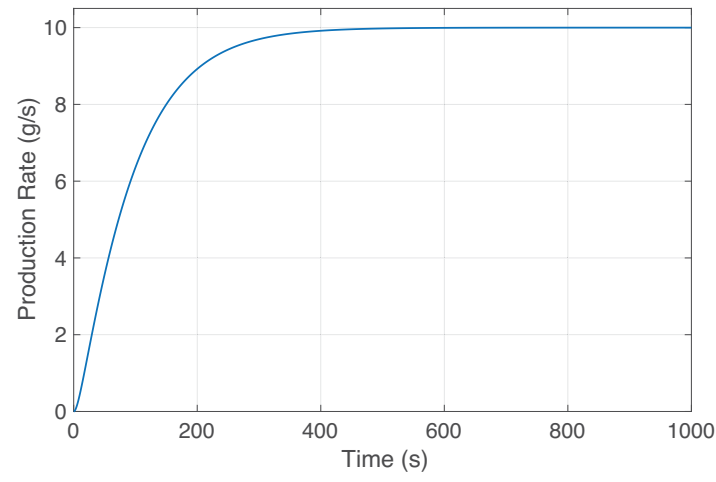

Figure 3. Output production rate of the mill for the defined parameters

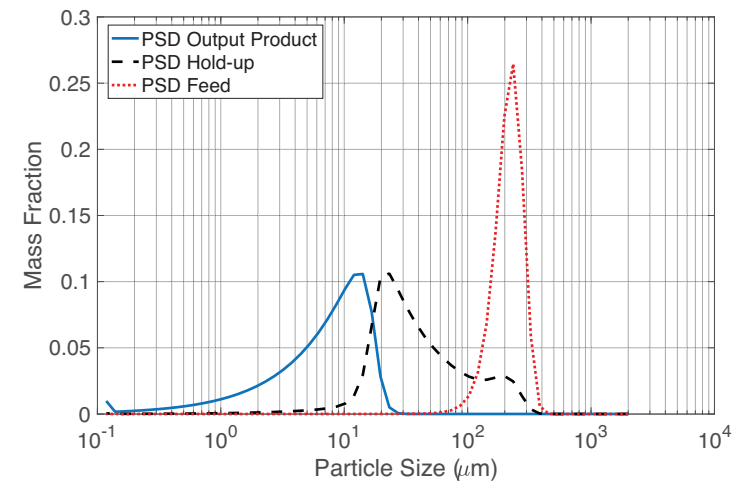

Figure 4. Particle Size Distribution of the output and the holdup of the mill and the feed used in the simulation

The feed PSD for the simulation is a Gaussian distribution with a mean of $200 \mu \mathrm{m}$ and a variance of 50 $\mu \mathrm{m}$ and is generated using the MATLAB command 'normpdf'. Figure 3. illustrates the evolution of the product output rate with time for the given parameter values. It can be observed after around $500 \mathrm{~s}$ the mill reaches a steady state. This type of dynamic behaviour is also observed on the real mill, albeit on different time scales. Figure 4. depicts the PSD of the output product as well as the PSD of the hold-up at the steady state condition. The shape of the PSD could be explained by the fact that given the structure of $P_{i}$, almost all the fine particles escape from the hold-up leading to the long shoulder towards the left in output PSD. Figure 5. shows evolution of the $\mathrm{D}_{10}, \mathrm{D}_{50}$, and $\mathrm{D}_{90}$ of the output product. The discrete jumps observed in the evolution of these variable is due to their numerical calculations from the cumulative PSD. Since, towards larger size end, the difference between the size classes is relatively larger that at lower end, the jumps are more exaggerated at higher values.

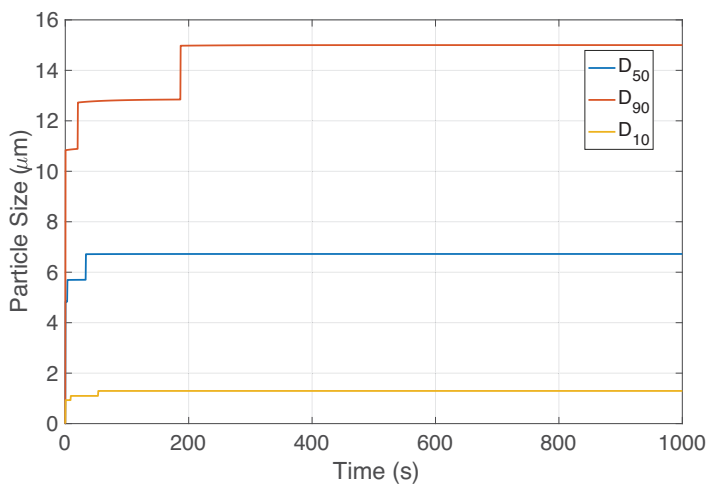

Figure 5. Evolution of particle size indicators $D_{10}, D_{50}, D_{90}$ of the output material with time

\section{Discussion}

Although the parameters considered in this model are not determined experimentally, it is worth noting that the model structure is able to capture dynamics of the mill which were observed during preliminary experimental analysis. The next step thus would be to estimate these parameters based on experimental data.

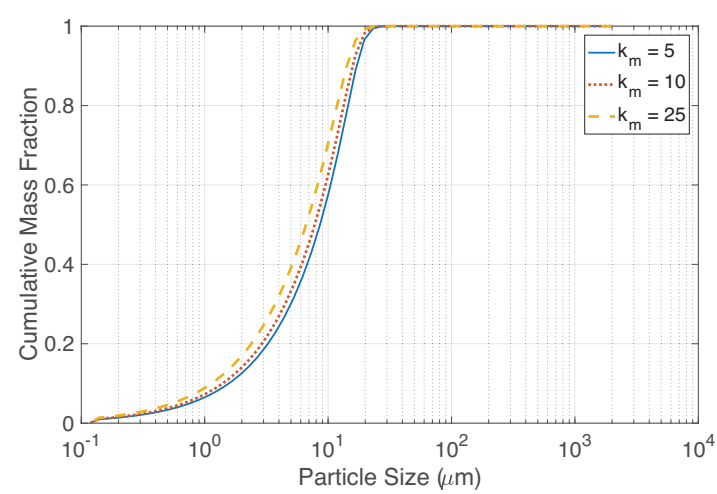

Figure 6. Cumulative particle size distribution of the output material for $\mathrm{k}_{\mathrm{m}}=5, \mathrm{k}_{\mathrm{m}}=10$, and $\mathrm{k}_{\mathrm{m}}=25$, with other parameter values constant

The parameter $\gamma$ in Equation 1 relates to the propensity of a material to break under loading conditions, while the parameter $\alpha$ determines the intensity of breakage which depends on the magnitude of the loading condition. The parameter $\gamma$ can be assumed to be constant for a particular API $[3,11]$. The parameter $\alpha$ on the other hand will be influenced by the operating conditions. Similarly, the parameters in the classification curve depend on the operating condition of the mill. This makes the parameter estimation for the model challenging as there will be a huge correlation between the breakage parameters and the classification parameters. It can be observed in Figure 6 that the effect of the parameter $\alpha$ on the output particle 
size is not as predominant as it is on the production rate and the stability of the mill as can be seen in Figure 7.

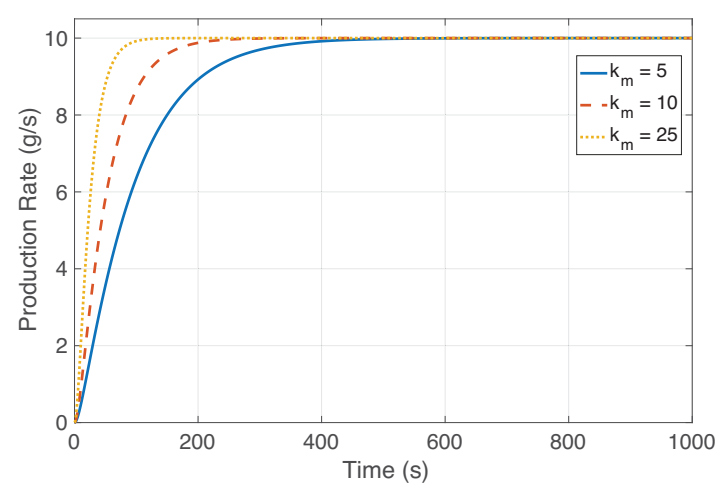

Figure 7. Output Flow Rate of milled material at $\mathrm{k}_{\mathrm{m}}=5, \mathrm{k}_{\mathrm{m}}=10$, and $\mathrm{k}_{\mathrm{m}}=25$

Figure 8 shows the output PSD of the milled material at different $x_{50}$ values. It can clearly be observed that this parameter has a more prominent effect on the output.

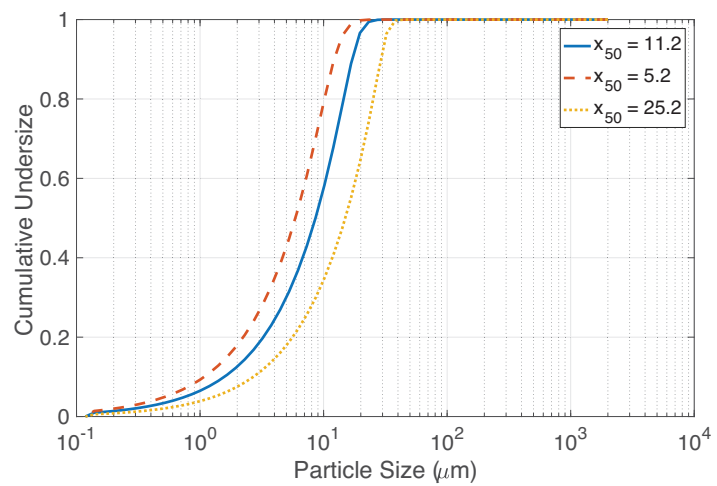

Figure 8. Output PSD of the milled material at $x_{50}=11.2, x_{50}=$ 5.2 and $x_{50}=25.2$ at other constant parameters

\section{Conclusions \& Future Work}

The model structure presented can capture the dynamics of a lab scale air jet mill. This model presents a simplification to a model proposed by Gommeren. The need for simplification arises from the fact that the parameters in the model are not directly identifiable from experimental data and not enough simulation studies were provided in the original study to get a comprehensive understanding of the model. However, the current model also runs into the similar difficulty as the Gommeren's model in that the breakage parameters are not directly identifiable from the measured data. Thus, at this stage a rigorous parameter identifiability analysis need to be carried out which should then be the basis for further model simplifications and experiment designs. The dependence of the breakage and classification parameters on the operating conditions also needs to be investigated. A priori, one can expect the breakage rate parameter, $\alpha$ to increase with increasing pressures and decrease with increasing feed rates. This is also reflected by the simulation shown in Figure 6. The effect on the classification parameters are more complicated. The classification parameters also have an effect on the PSD of the hold-up material inside the mill. This is to be expected as the classification function determines the residence time of a particle within the mill.

In future, other experimental techniques will be investigated to generate data which can be used to identify the breakage and classification parameters easily. Being in an industrial environment, it is important that the experimental time and the amount of materials required needs to be minimized. Hence, advance optimal experiment design techniques will be used to design experiments which can provide informational data which can be used to tune the model to any API that needs to be introduced. It is also envisioned, that the material dependent parameters may be linked to one or more API properties using a black box technique like artificial neural net. Ultimately, the goal of the research is to develop a model that can be easily tuned, and be implemented in a control framework such that given an API, a required PSD can be consistently delivered.

This research was supported by: KU Leuven project PFV/10/002 (OPTEC), project G.0930.13 of the Fund for Scientific Research-Flanders, and BelSPO Interuniversity Attraction Pole IAP VII/19 (DYSCO). SB holds a IWT Baekeland grant IWT150715 for his PhD research.

\section{References}

1. S. Teng, P. Wang, L. Zhu, M. W. Young, C. G. Gogos, Powder Technol. 195, 31 (2009)

2. F. Müller, R. Polke, G. Schädel, Int. J. Miner. Process. 44, 315 (1996)

3. H. J. C. Gommeren, D. A. Heitzmann, J. A. C. Moolenaar, B. Scarlett, Powder Technol. 108, 147, (2000)

4. S. Teng, P. Wang, L. Zhu, M. W. Young, C. G. Gogos, Chem. Eng. Sci. 65, 4323 (2010)

5. D. Starkey, C. Taylor, N. Morgan, K. Winston, S. Svoronos, J. Mecholsky, K. Powers, R. Iacocca, AIChE Journal, 60, 4086 (2014)

6. T. Brosh, H. Kalman, A. Levy, I. Peyro, F. Richard, Powder Technol. 257, 104 (2014)

7. Z. Zang, M. Ghadiri, Chem. Eng. Sci. 57, 3659 (2002)

8. D. Ramakrishna, Population Balances (Academic Press, San Diego, 2000)

9. M. Sen, R. Ramachandran, Adv. Powder Technol. 24, 51 (2013)

10. B. Epstein, Ind. Eng. Chem. 40, 2289 (1948)

11. L. Austin, Powder Technol. 5, 1 (1971)

12. H. Berthiaux, C. Varinot, J. A. Dodds, Chem. Eng. Sci. 51, 4509 (1996)

13. H. Otwinowski, Particul. Sci. Technol. 32, 399 (2014) 